\title{
The 2018 Lasker DeBakey Clinical Medical Research Award recognizes John Baird Glen for the discovery of propofol
}

\begin{abstract}
The Lasker DeBakey Award for Clinical Medical Research commends individuals whose achievements have profoundly advanced medicine. In 2018, the Albert and Mary Lasker Foundation honors John (Iain) Baird Glen, BVMS, PhD, for his contributions to the practice of anesthesia. Glen (Figure 1) spearheaded the discovery and clinical development of the i.v. anesthetic propofol, which has been identified among anesthesiologists as the most transformative drug in their field (1), as well as the development of a breakthrough infusion system that helped standardize anesthetic delivery. Together, propofol and its infusion system have remodeled expectations for the precision, safety, and tolerability of anesthesia.
\end{abstract}

The pursuit for total i.v. anesthesia

William Morton and others performed the first documented surgeries on anesthetized patients in the 1840s, and use of general anesthesia increased steadily throughout the remainder of the century. Early methods relied almost exclusively on gaseous inhalational anesthetics such as ether, chloroform, and nitrous oxide, which typically produced slow-onset anesthesia, sluggish recovery of consciousness, and - often - adverse postoperative effects (2).

Anesthetics (i.v.) possessed an important advantage over inhalational methods: they could provide a quicker onset of anesthesia. John Lundy, of the Mayo Clinic, is credited with bringing the i.v. anesthetic sodium thiopental into clinical practice in the 1930s. Injection (i.v.) of thiopental, a highly potent barbiturate, was found to induce anesthesia rapidly and displayed a favorable therapeutic index (3).

In spite of its advantages, thiopental was not considered suitable for maintaining anesthesia due to its sluggish metabo- lism. Multiple administrations dramatically increase patient recovery time and risk of adverse effects. Nonetheless, thiopental remained the preferred agent for anesthesia induction throughout the 1960 s, combined with newer inhalational agents for maintenance. The search for a compound that would enable total i.v. anesthesia continued (3).

\section{Glen's discovery of propofol}

Iain Glen's innovative career began with his studies of veterinary medicine at the University of Glasgow. After graduating in 1963, he spent a year as a veterinary surgeon at the University of Nairobi and returned with an interest in pursuing academic research rather than veterinary practice. In 1965, he accepted a lecturer position in the Department of Small Animal Surgery at his alma mater, an opportunity that would also allow him to conduct research in veterinary medicine. To facilitate his teaching role, he studied for the University of Glasgow Royal College of Veterinary Surgeons' newly created specialty diploma in Veterinary Anesthesia. The training, which qualified him to teach the topic, also ultimately redirected his lifelong research interests.

Glen became particularly interested in investigating several newly developed anesthesia agents, including the steroid mixture of alfaxalone and alfadolone and the sedatives xylazine and droperidol. Eventually, Glen changed the focus of his $\mathrm{PhD}$ to studies on the pharmacology of injectable anesthetic agents.

In 1972, Glen joined ICI Pharmaceuticals' anesthetics research team, which sought to identify a compound that matched thiopental's induction speed but improved upon its slow metabolism. Although ICI's chemists were producing new compounds with structures that resembled known anesthetics, Glen was paired with chemist Roger
James, who thought it was worthwhile to revisit ICI's existing compound collection.

The older arsenal of compounds had been abandoned because of poor solubility in water, a requirement for i.v. injection.

"It's a bit of a catch-22 situation," explains Glen. "You need a lipid-soluble molecule to get it into the brain, but if you have a lipid-soluble molecule, you can't get it into aqueous solution."

Auspiciously, the steroid anesthetics Glen had studied at the University of Glasgow had been formulated in a surfactant material known as Cremophor EL, which made it possible to solubilize lipid molecules in an aqueous solution. Glen and James identified anesthetic activity in 2,6 diethylphenol, but it was not sufficiently potent or rapidly acting enough to compete with thiopental. However, the observation turned their attention toward an array of similar hindered phenol compounds. Among the first three compounds tested was 2,6 diisopropylphenol (propofol). Although the chemical effort continued until 1976 and evaluated 300 phenols in total, no compound with superior properties to propofol was discovered. Over the duration of the project, more than 3,000 candidates were tested for anesthetic activity.

\section{Early challenges}

Propofol and thiopental had similar therapeutic indices, but propofol was more potent and carried a much lower risk of postoperative nausea. Due to its speedy metabolism, propofol also retained its short recovery latency throughout repeated dosing, which represented a clear advantage over thiopental (4).

Still, Glen tells the JCI, "It was quite a struggle to convince the company that propofol was worth developing."

ICI Pharmaceuticals' forecasts for propofol's commercial potential were pessimistic, but Glen was convinced of the compound's promise.

"I had to go and speak to the technical director and try to explain to him that this 


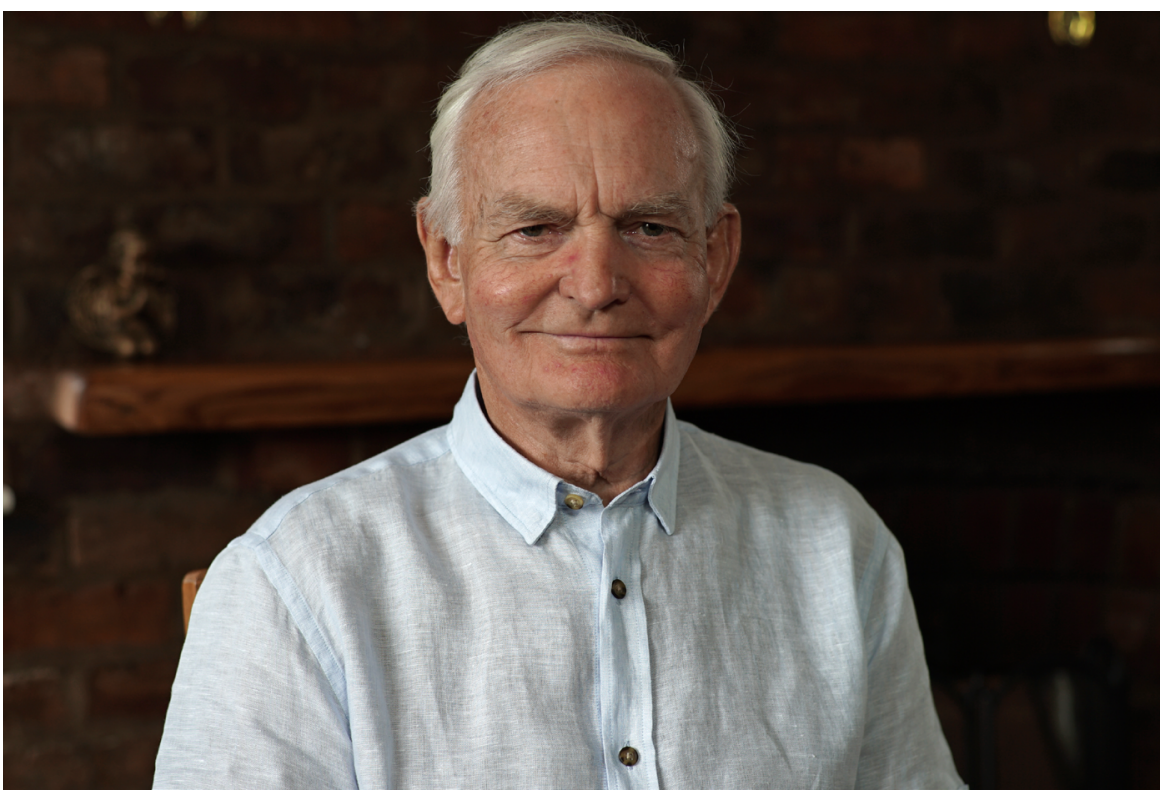

Figure 1. Iain Glen, recipient of the Lasker DeBakey Award for Clinical Medicine Research. Image credit: Flora Lichtman.

was not just another thiopental. This was a drug which could be used much more widely because of its rapid metabolism," he says.

He convinced the company that propofol could be appropriate for a greater variety of therapeutic applications than thiopental, including maintenance of anesthesia, sedation in intensive care patients, and sedation in conjunction with regional or local anesthetic techniques.

In initial trials, propofol was solubilized with Cremophor EL, but the formulation was not ideal. Glen and colleagues conducted a series of experiments implicating Cremophor EL as responsible for the anaphylactoid reactions associated with alfaxalone, alfadolone, and other agents. They subsequently spent 2 years searching for an alternative surfactant formulation suitable for clinical use before shifting their focus to emulsions. Ultimately, they developed a soybean oil formulation to emulsify propofol and render the product fit for clinical trials (5).

\section{Clinical development of propofol}

Clinical trials had initially been performed in Belgium with the Cremophor EL-based formulation. The results were somewhat chaotic, plagued by inconsistent administration and inadequate records.

"Some of the early results really were quite confusing," Glen says. "I can remem- ber one meeting when, by 5 votes to 4 , we decided to continue development. There was a need to fight for the drug to that extent because it wasn't widely supported by all parties in the development team." Faced with waning company enthusiasm, Glen was an advocate for propofol's improved emulsion formulation.

Glen transferred from research to the medical affairs department in 1983 to assist Ron Stark and Katie Hopkins in the clinical evaluation of the emulsion formulation. He was particularly interested in advancing propofol's use for maintenance of anesthesia in specialized patient groups in whom inhalational anesthesia was particularly inconvenient or life-threatening, including patients undergoing operations on the head, neck, or airway and those susceptible to malignant hyperthermia.

The ensuing clinical trials were successful, and propofol entered the United Kingdom's clinical market as Diprivan in 1986. By this time, commercially available technologies made it possible to conduct the continuous, real-time physiologic monitoring that is now standard during anesthesia and recovery periods. Pharmacokinetic modeling, which had been employed with increasing precision since the 1960s, was also refining the guidelines for dosing patients safely and effectively.

Academic researchers were beginning to utilize pharmacokinetic models to develop computer algorithms for optimal anesthetic administration, and Glen saw a new opportunity to improve propofol's application in the surgical setting. He organized a meeting between research groups from all over the world to determine whether ICI should develop a modeldriven device for propofol.

"At the end of that meeting, I was convinced that target-controlled infusion, or TCI as it's called, would make the adoption of maintenance of intravenous anesthesia easier. It took me another couple of years to convince ICI to begin development in that area," he says.

Working with Gavin Kenney and Martin White, Glen oversaw the development and clinical evaluation of a TCI system that relied on propofol's pharmacokinetics to safely induce and maintain anesthesia. The Diprifusor TCI module could be installed in any compatible commercially available syringe pump, ensuring consistent performance across surgical settings (6). Coordinated commercial marketing of Diprivan and Diprifusor began 1996, and both remain in wide use globally.

\section{The legacy of Glen's innovations}

Iain Glen's predictions for propofol's widespread applications were correct: Today, propofol remains the preferred anesthetic in numerous diverse medical procedures. Though he notes that the drug has drawbacks - it can cause injection-site pain in some patients, and the original emulsion formulation was susceptible to bacterial contamination - Glen is pleased with propofol's success.

"It's a credit to the drug. As far as the quality of recovery is concerned, I think it's fantastic that patients who experience propofol anesthesia do actually see the difference if they have had an earlier anesthetic with thiopentone," he says.

Glen is also optimistic that propofol will produce further advancements in anesthetic practice. The Diprifusor system was never approved in the United States, but he is hopeful that a commensurate device may make it to market now that propofol is available as a generic drug. Ongoing research into the drug's pharmacokinetics, specifically in pediatric patients, continues to improve anesthetic methods and patient outcomes (7). 
Glen says that receiving the Lasker DeBakey award is a "huge honor and a great surprise. I believe serendipity played a role in the initial discovery. I knew what I was looking for, and it was largely a matter of persevering and overcoming a number of barriers to get the drug to the market. It's the drug really, I think, that deserves the award."

In propofol, Glen recognized an agent whose properties were capable of revolutionizing anesthetic practice. His persistent push for propofol's clinical development and expansion into multiple therapeutic indications were critical to actualizing innovations that have dramatically improved general anesthesia for the millions of patients who undergo surgery each year. For these transformative contributions to anesthesiology, the Lasker Foundation presents Dr. Iain Glen with the 2018 Lasker DeBakey Award for Clinical Medicine Research.

\section{Elyse Dankoski}

1. Bateman BT, Kesselheim AS. Propofol as a transformative drug in anesthesia: insights from key early investigators. Drug Discov Today. 2015;20(8):1012-1017.

2. Webster NR, Galley HF, eds. Landmark Papers in
Anaesthesia. Oxford, United Kingdom: Oxford University Press; 2013.

3. Corssen G. John S. Lundy: Father of intravenous anesthesia. In: Rupreht J, van Lieburg MJ, Lee JA, Erdmann W, eds. Anaesthesia. Berlin, Heidelberg, Germany: Springer; 1985.

4. Glen JB. Animal studies of the anaesthetic activity of ICI 35 868. Br J Anaesth. 1980;52(8):731-742.

5. Glen JB, Hunter SC. Pharmacology of an emulsion formulation of ICI 35 868. Br J Anaesth. 1984;56(6):617-626.

6. Glen JB. The development of 'Diprifusor': a TCI system for propofol. Anaesthesia. 1998; 53(suppl 1):13-21.

7. Mahmoud M, Mason KP. Recent advances in intravenous anesthesia and anesthetics. F1000Res. 2018;7(F1000 Faculty Rev):470. 